\title{
INVESTIGACIONES
}

\section{Desafíos a la formación inicial docente en convivencia escolar ${ }^{*}$}

\author{
Challenges to initial teacher training in school coexistence
}

\author{
Laurent Loubiès Valdés ${ }^{a}$, Pablo Valdivieso Tocornal ${ }^{a}$, Catalina Vásquez Olguín ${ }^{a}$ \\ ${ }^{a}$ Equipo de Convivencia Escolar, Universidad de Chile. \\ lloubiesv@gmail.com, pvaldivieso@uchile.cl, catalina.vasquez@ug.uchile.cl
}

\begin{abstract}
RESUMEN
La formación inicial docente es clave para articular políticas educativas de convivencia escolar y ejercicio profesional docente. Esta investigación buscó identificar los desafíos que enfrenta la formación inicial docente chilena en el ámbito de la convivencia escolar. Metodológicamente, se entrevistó en profundidad a formadores de formadores de distintos niveles del sistema y se llevó a cabo un análisis cualitativo de contenido de la información recabada. Los resultados sugieren que la convivencia escolar sea incorporada de manera relevante en el currículum de la formación inicial docente. Los desafíos formativos que surgen son: herramientas conceptuales en convivencia escolar, gestión de la diversidad, abordaje de maltrato, violencia y conflicto, manejo de clima de aula, habilidades socioemocionales y trabajo colaborativo. Se proponen estrategias curriculares para la implementación y transformaciones culturales necesarias para sustentar el proceso. Se espera que estos desafíos contribuyan al desarrollo de políticas públicas y propuestas formativas concretas para el ámbito.
\end{abstract}

Palabras claves: Formación de profesores, aprender a vivir juntos, relaciones en la escuela, formadores de formadores.

\section{ABSTRACT}

Initial teacher training is considered as a key instance to articulate the educational policies of school coexistence with the professional teaching practice. This research aimed to identify the challenges faced by Chilean initial teacher training in the field of school coexistence. Methodologically, teacher educators from different levels of the system were interviewed in depth and a qualitative analysis of the content of the collected information was carried out. The results suggest the incorporation of school coexistence as a relevant area in the curriculum of the initial teacher training. The formative challenges that arise are: conceptual development in school coexistence, diversity and education, mistreatment, violence and conflict approach, classroom climate, socio-emotional skills and collaborative work. Curricular strategies for the implementation and cultural transformations necessary to sustain the process are proposed. It is expected that these challenges contribute to the development of public policies and concrete training proposals for the field.

Key words: Teacher training, learning to live together, relationships at school, teacher educators.

\footnotetext{
* Este trabajo fue financiado a través del Proyecto n²2162356 del año 2016 del Programa de Formación de Capital Humano
} Avanzado "Becas Magíster Nacional" de CONICYT. 


\section{INTRODUCCIÓN}

El "aprender a vivir juntos" constituye uno de los principales objetivos de la educación contemporánea (Delors, 1996). Por eso, en Chile, se ha procurado transitar hacia un nuevo paradigma en cuanto a los objetivos formativos de la escuela, extendiéndose estos más allá de la formación por disciplinas e incorporando las nociones de formación integral y los Objetivos de Aprendizaje Transversales de la educación (Ministerio de Educación o MINEDUC, 2009; 2013). Así, en las últimas décadas, han cobrado relevancias temáticas tales como la calidad de las relaciones interpersonales, la aceptación de la diversidad, la convivencia democrática, el enfrentamiento y resolución de conflictos y el abordaje de las dinámicas de violencia escolar, entre otras competencias psicosociales a ser desarrolladas en la escuela (Toledo, 2018). Estas temáticas se han institucionalizado progresivamente en torno al concepto general de Convivencia Escolar (en adelante, $\mathrm{CE}$ ), generándose un conjunto de políticas, orientaciones y marcos normativos al respecto (MINEDUC, 2002, 2003, 2015, 2019; Valdivieso, González, Toledo y Leyton, 2017).

Paralelamente, además de la ampliación de sus objetivos, el sistema educativo ha tenido transformaciones tales como la extensión de su cobertura a segmentos históricamente excluidos, el fortalecimiento de demandas sociales por calidad y democratización de la educación y la visibilización de distintas formas de maltrato y violencia en el medio escolar, lo que ha tenido como resultado la complejización del trabajo docente (Cornejo, 2008; Canales, Bellei y Orellana, 2016).

En este contexto, se considera la Formación Inicial Docente (en adelante, FID) como un subsistema clave para vincular estratégicamente la profesión docente con los desafíos educativos contemporáneos. No obstante, los propios docentes han colocado en entredicho la capacidad de la FID para prepararlos para abordar la dimensión convivencial del proceso educativo (Gaete, Gómez y Bascopé, 2016). Pareciera que la formación en esta área no ha sido llevada a cabo ni investigada de manera consistente y sistemática en Chile (Manzi, 2010; Cisternas, 2011).

Una reciente línea de investigación ha procurado explorar los abordajes en FID en CE, visibilizando debilidades acusadas tanto por el magisterio en ejercicio como al interior de los procesos formativos iniciales (Ruffinelli, 2013; Canedo, 2014; Leyton, 2014; Ferrada, Villena y Turra, 2015; Gaete, Gómez y Bascopé, 2016). Frente a esta constatación, surge la interrogante que guía la presente investigación: ¿A qué desafíos se enfrenta actualmente la FID chilena en materia de desarrollo de competencias docentes para la CE? Se espera que los hallazgos sirvan como insumos para nutrir los procesos reflexivos y de innovación al interior las casas de estudio en que los docentes se preparan.

\section{MARCO REFERENCIAL}

\subsection{LA CONVIVENCIA ESCOLAR COMO DIMENSIÓN RELEVANTE EN EDUCACIÓN}

El concepto de CE incorpora el conjunto de dinámicas relacionales que se establecen al interior de una comunidad educativa. Una buena $\mathrm{CE}$ se ha definido legalmente como la "coexistencia armónica de los miembros de la comunidad educativa, que supone una interrelación positiva entre ellos y permite el adecuado cumplimiento de los objetivos educativos en un clima que propicia el desarrollo integral de los estudiantes" (Ley 
$\mathrm{N}^{\circ} 20.536,2011$ ). Por su parte, la Política Nacional de Convivencia Escolar (en adelante, PNCE) vigente entre 2015 y 2018 (MINEDUC, 2015) señalaba que el concepto supera el carácter instrumental de las normas y rutinas escolares, apuntando a una visión más integral: La CE es un proceso que potencia la formación ciudadana. Asimismo, la actual PNCE plantea que ésta "se centra en dos ámbitos fundamentales: por un lado, en el sentido formativo y ético de la CE; por otro, en la idea central de que todos los miembros de la comunidad educativa estamos llamados a ser responsables y beneficiarios de una buena convivencia" (MINEDUC, 2019).

La política educacional chilena adoptó el término "Convivencia Escolar" haciendo eco del consenso internacional plasmado por la UNESCO en torno a la importancia de "aprender a vivir juntos" como objetivo de la escuela (Valdivieso et al., 2017). Magendzo, Toledo y Gutiérrez (2013) profundizan esta idea visibilizando su contexto específico: ciertos países esperan que sus sistemas educativos contribuyan activamente a prevenir experiencias de violencia social y política extremas como la guerra o en el caso de Chile, la dictadura.

El enfoque formativo de la CE apela a la enseñanza y aprendizaje de conocimientos, habilidades, valores y principios que permiten vivir en paz y armonía con otros y prevenir así la violencia (Sandoval, 2014). De acuerdo con la mencionada PNCE 2015-2018, en coherencia con su mirada integral y de formación ciudadana, a dicho enfoque formativo deben sumarse otros como los enfoques de derecho, de género, de gestión territorial, de participación y de inclusión y de gestión institucional. No obstante, Valdivieso et al. (2017) constatan que a pesar de que estos enfoques están declarados en las políticas públicas, todavía predomina en las prácticas pedagógicas y de gestión escolar, una lógica instrumental de la CE, en la medida que ésta se concibe, con mayor énfasis, como una dimensión para posibilitar aprendizajes medidos por pruebas estandarizadas, más que como un fin educativo en sí mismo.

Investigadores como Ahumada y Tapia (2013) y Valdivieso et al. (2017) han rastreado las iniciativas emprendidas por el MINEDUC y/o plasmadas en la legislación chilena para institucionalizar progresivamente la responsabilidad del sistema escolar en relación con la CE: Los valores del "aprender a vivir juntos" se consagraron como objetivos explícitos de aprendizaje en el aula bajo la forma de Objetivos Fundamentales Transversales y luego de Objetivos de Aprendizaje Transversales (MINEDUC, 2012). Por otra parte, sucesivas iniciativas legales han elevado la CE a la categoría de desafío institucional al incluirla como dimensión de la gestión educacional (Ruz, 2006). Ejemplo de ello son las sucesivas PNCE (MINEDUC, 2002; 2015; 2019), los criterios del Sistema de Aseguramiento de la Calidad de la Gestión Escolar (MINEDUC, 2006), la Ley sobre Violencia Escolar (Ley $\left.\mathrm{N}^{\circ} 20.536,2011\right)$ y más recientemente, los Indicadores de Desarrollo Personal y Social (Decreto Supremo N’381, 2013).

Cabe acotar que, si bien las normativas definen la CE como responsabilidad de todos los estamentos de las comunidades educativas, Carrasco, López y Estay (2012) puntualizan que la responsabilidad formal recaería principalmente, en virtud de la Ley sobre Violencia Escolar, en el personal contratado por los establecimientos, es decir, principalmente, en directivos, docentes y asistentes de la educación. En coherencia con este análisis, ya desde la elaboración del Marco para la Buena Enseñanza o MBE (MINEDUC, 2003), se establece como competencia docente el favorecer "climas propicios al aprendizaje" en las aulas, caracterizados por el respeto, la empatía, la seguridad, la equidad y la solidaridad. 
Otros autores también coinciden en asignar un rol preponderante al docente en la materia (Milicic y Arón, 2000; Mena y Valdés, 2008; Sandoval, 2014).

Por otro lado, López et al. (2011) han alertado respecto de la tendencia discursiva en los establecimientos a responsabilizar a los estudiantes de sus problemas de conducta y violencia, reproduciendo ciclos de exclusión escolar; proponen como solución una revisión de las visiones, retóricas y prácticas que sustentan dicha exclusión, cuestionando asimismo la función social de la escuela.

Arístegui et al. (2005) y Ruz (2006) abordan esta idea al plantear la necesidad de que la pedagogía, como disciplina en sí, propicie nuevos modelos de docencia centrados en la CE como propuesta valórica, formativa y transformadora. Sugieren que la creación de una "pedagogía de la convivencia" implicaría un cambio de los modelos pedagógicos tradicionales y un distanciamiento de las prácticas hegemónicas. A su juicio, una pedagogía de la convivencia debería entenderse como un cruce de caminos que enfaticen el carácter social e intersubjetivo de la relación pedagógica, las dimensiones transversales del currículum y conceptos como el conflicto, la realidad social y la diversidad.

García (2008) propone líneas temáticas para abordar la CE desde una perspectiva pedagógica, las llama "respuestas pedagógicas", no porque pertenezcan exclusivamente al rol docente o al contexto escolar formal, sino por poseer una "lógica pedagógica". Estas respuestas consisten en favorecer la inclusión y el aprendizaje de la diversidad, educar en valores, promover la mediación como forma privilegiada de resolver de manera dialogante y constructiva los conflictos, recuperar el diálogo entre la escuela y la familia, promover la participación real de todos los miembros de la comunidad educativa en la toma de decisiones, definir derechos, deberes y normas de convivencia y mantener un permanente diálogo entre la escuela y el mundo social en que se inscribe.

A partir de dicha propuesta, Canedo (2014) organiza dimensiones que el concepto de CE podría abarcar como materia de interés para los docentes: comprendería los valores democráticos, el manejo de los conflictos, la ciudadanía y la participación. Incorpora también la categoría "desarrollo socio-afectivo" para integrar el desarrollo emocional y las habilidades socio-emocionales.

\subsection{LA FORMACIÓN INICIAL DOCENTE}

La FID es el proceso de enseñanza y aprendizaje en torno a un saber específico y estandarizado, llevado a cabo en instituciones especializadas para ello durante un período regulado y que termina con la certificación de competencias docentes (Cox y Gysling, 2009). Las actividades curriculares que la componen, ya sean cursos, seminarios o talleres se dividen en tres grandes áreas de formación: la general, orientada a favorecer la cultura general y el conocimiento del sistema educativo, la profesional o pedagógica, que agrupa las asignaturas sobre teorías o procedimientos directamente vinculados al proceso de educar y la disciplinar o de especialidad, que reúne los conocimientos propios de una materia determinada y que constituirá el área de especialización del docente (Cox y Gysling, 2009; Ávalos, 2014; Leyton, 2014). Existe un menor consenso para identificar la formación práctica o formación profesional aplicada como un área distinta de las anteriores. Ésta considera las actividades de contacto con las escuelas y aulas, pre-prácticas y prácticas profesionales (Ávalos, 2014; Canedo, 2014), pero se la puede considerar parte de la formación profesional (Cox y Gysling, 2009). 
Cabe señalar que la FID no sólo lleva al desarrollo de competencias profesionales específicas y acreditables formalmente, sino que también produce una identidad profesional. Ésta se constituye tanto por las competencias adquiridas como por la manera en que la profesión docente y las instituciones que la forman se posicionan en la sociedad (Cox y Gysling, 2009). A ese respecto, se puede señalar que las ideas, prácticas y expectativas que caracterizan a quienes se desempeñan formando docentes también influyen en la construcción de dicha identidad profesional (Reyes-Jedlicki et al., 2014).

El más reciente mecanismo de mejoramiento y aseguramiento de la calidad de la FID es el progresivo desarrollo de estándares de contenido para las carreras de Pedagogía, llevado a cabo en el marco del proceso de acreditación obligatoria (CNA Chile, 2018). Anteriormente, en 2002, el Programa gubernamental de Fortalecimiento de la Formación Inicial Docente o FFID había concluido proponiendo un sistema de evaluación de la calidad de la FID aplicable a estudiantes de Pedagogía a punto de egresar, basado en estándares de desempeño (MINEDUC, 2000; citado en Ávalos, 2004). Dichos estándares, relativos al "saber hacer" docente se organizaban en torno a las cuatro dimensiones que más tarde constituyeron los dominios del MBE (Baeza, Pérez y Reyes, 2006). A partir de entonces, el Centro de Perfeccionamiento, Experimentación e Investigaciones Pedagógicas o CPEIP del MINEDUC ha trabajado en la confección de propuestas de "Estándares Orientadores" para las carreras de Pedagogía en sus distintos niveles, especialidades y menciones, apareciendo las primeras en 2013. Éstos refieren principalmente a contenidos disciplinares y pedagógicos, incluyendo además referentes didácticos para el contenido disciplinar (Ávalos, 2014).

Cisternas (2011) señala como desafíos todavía pendientes la articulación de la formación pedagógica con la de especialidad, el aprendizaje de contenidos curriculares, la relación entre la formación y el conjunto del sistema educativo y el desarrollo de procedimientos de monitoreo y evaluación de los programas.

En lo que respecta al desempeño docente esperado en CE, ha sido descrito de manera incipiente en las últimas dos décadas como dimensión para su estandarización en el MBE, documento que en la práctica constituye un referente para todos los demás procesos de estandarización (Sotomayor y Gysling, 2011; Ávalos, 2014). Se espera que los docentes sean capaces de crear un ambiente propicio para el aprendizaje, en términos de clima de aula, establecimiento de normas y ambiente organizado de trabajo (MBE, 2003). No obstante, a pesar de nombrarse en el MBE y los documentos que de él emanan, la CE no ha sido foco de atención particular de las políticas de mejora de la FID.

\subsection{HACIA UNA FORMACIÓN INICIAL DOCENTE EN CONVIVENCIA ESCOLAR}

Durante los últimos años, la academia se ha interesado progresivamente por la preparación que reciben los docentes para hacerse cargo de la CE.

Ruffinelli (2013) indagó en la evaluación de docentes nóveles sobre sus propias experiencias de formación universitaria. Los encuestados coincidieron en evaluar peor la formación en temáticas como el trabajo con las familias, el manejo de la disciplina en el aula, la comprensión del sistema educacional y la atención a la diversidad, entre otras. Gaete, Gómez y Bascopé (2016) ratificaron esta idea en una investigación con docentes más experimentados. Según ellos, las insuficiencias formativas se relacionarían con la atención a la diversidad, el dominio de grupo y de la relación con apoderados, entre otras áreas. 
Por otro lado, Leyton (2014) y Canedo (2014), pertenecientes a un mismo equipo de investigación, exploraron los currículum declarados de dos universidades chilenas formadoras de docentes para identificar temáticas alusivas a la CE en sus programas de Pedagogía. Por medio del estudio de mallas curriculares, programas de asignaturas y perfiles de egreso, evidenciaron la escasa presencia del concepto CE, de dimensiones de la misma o de ideas afines. No obstante, lograron categorizar las alusiones a conceptos y dimensiones de la CE en la FID, distinguiendo entre,

1) realidad sociocultural de la escuela, referente a cultura escolar, clima escolar, conflicto y disciplina,

2) políticas educativas y transversalidad,

3) valores democráticos, que consideran respeto, diálogo, tolerancia, justicia, paz, solidaridad, empatía, cooperación, solidaridad y responsabilidad,

4) desarrollo socio-afectivo, que engloba el despliegue de la afectividad y las habilidades sociales y emocionales y

5) formación ciudadana, que busca potenciar la formación de sujetos activos de una sociedad democrática, considerando los conceptos de democracia, ciudadanía, derecho, identidad, familia, comunidad educativa, diversidad, inclusión y multiculturalidad.

Pese al esfuerzo de los autores por categorizar y rastrear los contenidos aludidos, concluyeron que la FID en CE es muy acotada, precaria y superficial. Esta conclusión es coherente con la percepción de insuficiencia en la formación expresada por los docentes según las investigaciones citadas más arriba.

Finalmente, cabe hacer referencia a la investigación internacional sobre innovaciones del currículum de la FID para la incorporación de las dimensiones de la CE. Los países respecto de los que se encontró investigación científica actualizada fueron Colombia, Brasil y España. Es particularmente relevante destacar en las investigaciones revisadas la presencia de una reflexión explícita respecto del sentido ético y político de los procesos de modernización curricular que han emprendido para cada contexto. En Colombia, se ha propuesto aumentar oportunidades de colaboración, diálogo y reflexión entre docentes en formación para el desarrollo de principios vinculados a la justicia social (Sierra, 2016). En Brasil, la modernización de prácticas pedagógicas ha pretendido desincentivar la aparición de violencia escolar (Gomes \& Pereira, 2009). En la experiencia de España, se ha promovido el desarrollo del pensamiento pedagógico e incluso de la identidad profesional del magisterio con miras a la formación de un perfil de maestros para la democracia (Pérez, 2018).

\section{DISEÑO DE INVESTIGACIÓN}

\subsection{OBJETIVOS}

La presente investigación tuvo como objetivo identificar los desafíos que enfrenta la FID en el ámbito de la CE, en este caso, desde la perspectiva de los formadores de formadores. El concepto de desafío se entiende como la tarea pendiente que se define al constatar la brecha entre un diagnóstico actual y una situación deseada (Miranda y Rivera, 2009; Contreras, 2014; Gaete, Gómez y Bascopé, 2016; Gómez, 2016). 


\subsection{METODOLOGÍA}

La investigación se desarrolló desde un enfoque metodológico cualitativo, con el fin de acceder al objeto de estudio desde los marcos de referencia que los propios entrevistados manejan en calidad de expertos (Taylor y Bogdan, 2000; Strauss y Corbin, 2002).

Se eligieron como informantes claves a los formadores de formadores, aquel colectivo profesional que media la construcción de conocimientos de futuros docentes (Alvarado, 2006; citado en Reyes-Jedlicki et al., 2014). Éstos desempeñan su actividad formativa mediante acciones diversas como la definición y ejecución de programas de formación, la realización de clases en licenciaturas, pedagogías, habilitaciones pedagógicas, posgrado o extensión. También realizan investigación educacional (incluyendo la formación de tesistas), coordinación de prácticas profesionales y colaboración en el desarrollo profesional docente desde la escuela, como mentores o guías de práctica (Ávalos, 2004; Cisternas 2011; ReyesJedlicki et al., 2014). Por lo mismo, se consideraron tres niveles según el lugar y grado de influencia que ocupan en el sistema de FID:

El nivel sistémico (A) que engloba a quienes diseñan y discuten las políticas públicas de FID e influyen directamente en la elaboración de directrices para el sistema. Incluye a miembros de organismos de alcance nacional encargados del diseño de la política o de centros de pensamiento e investigación. Se entrevistaron 4 sujetos en este nivel. El nivel universitario (B) considera a académicos de las carreras de Pedagogía. Incluye directivos, docentes de asignaturas y coordinadores de prácticas profesionales. Se entrevistaron 6 sujetos en este nivel. El nivel de terreno (C) incluye a docentes que trabajan en establecimientos escolares y que además cumplen el rol de tutores para estudiantes de Pedagogía en etapa de práctica profesional. Se entrevistaron 4 sujetos en este nivel. Para los niveles $\mathrm{B}$ y $\mathrm{C}$ que no son de alcance sistémico, se consideraron dos universidades públicas de la Región Metropolitana, caracterizadas por una larga y reconocida trayectoria en FID. Los participantes fueron contactados mediante un muestreo de tipo "bola de nieve" (Taylor y Bogdan, 2000).

En definitiva, la muestra entrevistada quedó conformada por 14 sujetos: nueve mujeres y cinco varones. Las entrevistas fueron realizadas entre el segundo semestre de 2017 y primer semestre de 2018.

La técnica utilizada para producción de la información fue la entrevista en profundidad. En concreto, se elaboró una pauta especialmente para la entrevista. La pauta definitiva consideró matices y especificaciones menores para los distintos niveles, manteniendo estructura y objetivos comunes. Se indagó respecto de los desafíos en CE que enfrentan los docentes en ejercicio en el medio escolar y respecto de los desafíos que éstos plantean a la FID. La información recabada, registrada y transcrita fue sometida al análisis de contenido de carácter cualitativo descrito por Taylor y Bogdan (2000).

\section{RESULTADOS}

Los resultados se presentan organizados en tres categorías principales: En primer lugar, se describen los desafíos al ejercicio docente que los entrevistados valoran como indispensables de incluir en los procesos formativos iniciales. En segundo lugar, se describen las estrategias curriculares que serían necesarias para la incorporación de los 
desafíos formativos propuestos. En tercer lugar, se señalan las transformaciones culturales que se deberían impulsar para dar sustento al proceso de FID en CE.

\subsection{DESAFÍOS FORMATIVOS EN CONVIVENCIA ESCOLAR}

Esta primera gran categoría agrupa el conjunto de habilidades, contenidos, valores y actitudes directamente vinculados a la $\mathrm{CE}$ que los entrevistados ven como indispensables para incorporar en el currículum de las Pedagogías.

\subsubsection{Herramientas conceptuales en Convivencia Escolar}

El área de formación pedagógica debe abordar los desarrollos conceptuales sobre CE, con relación a los cuales los futuros docentes deberían poseer un alto grado de actualización, dado el acelerado avance científico en la materia y la importancia progresiva que ésta adquiere en las políticas públicas educacionales.

Al momento de explicitar esta categoría, los entrevistados enumeran temáticas como las conductas disruptivas, la discriminación, las peleas y el bullying y la existencia de una PNCE que se actualiza periódicamente. No obstante, surge un énfasis en la fuerte vinculación entre el estudio y el abordaje práctico de la CE y la psicología como ciencia. Esto porque consideran que el conocimiento del desarrollo psicológico o psicosocial escolar permite comprender la etapa vital, las motivaciones, las conductas y las maneras de relacionarse propias de los estudiantes. Su estudio proveería una comprensión de cómo los escolares se desenvuelven tanto en contexto de aula regular como en coyunturas caracterizadas por fenómenos sociales como la organización y la movilización política estudiantil.

\subsubsection{Gestión pedagógica de la diversidad escolar}

Un segundo desafío perfilado es el de preparar competencias o habilidades para una gestión pedagógica de la creciente diversidad estudiantil que caracteriza el aula de hoy, de manera que sus decisiones profesionales sean pertinentes e inclusivas.

La ampliación de la cobertura educativa hacia los sectores y segmentos más vulnerables, así como la incorporación y la permanencia en el sistema de un estudiantado cada vez más diverso, constituyen para los entrevistados un sello de las políticas públicas que se mantendrá como tendencia. La diversidad escolar se expresa en la dimensión socioeconómica, en las pautas de comportamiento de los estudiantes, en sus capacidades intelectuales y de aprendizaje, en su manejo de contenidos, en sus capitales culturales particulares, en su manera de afrontar el conflicto, en la diversidad de culturas a las que pertenecen y en las necesidades educativas especiales que presentan.

\subsubsection{Abordaje del maltrato escolar, la violencia y el conflicto}

Otro desafío que surge es favorecer la capacidad de intervenir pedagógicamente en un contexto en que la violencia se encuentra normalizada y naturalizada, considerada casi como un código compartido por los estudiantes para relacionarse y resolver conflictos.

Los entrevistados ven las peleas entre escolares como una interrupción al normal desarrollo de las actividades pedagógicas. No obstante, también ponen de manifiesto que 
los estudiantes son, a su vez, víctimas de violencia en sus casas o barrios, las que se suman a las violencias presentes en el medio escolar. Incluso, se reconoce que los estudiantes no son los únicos protagonistas de la violencia escolar ni sus únicas víctimas en la medida en que los establecimientos alojan muchas veces maltratos dentro de y entre los estamentos que conforman las comunidades escolares.

\subsubsection{Manejo del clima de aula como condición de enseñanza}

Se señala que el docente debe ser capaz de manejar el comportamiento de los estudiantes porque la disrupción se puede entender como una amenaza al clima de aula y un obstáculo para el desarrollo de la clase. Se asume que sólo se pueden llevar a cabo las actividades planificadas en un clima de tranquilidad y sin maltrato. Crear un clima de aula favorable se vuelve un esfuerzo permanente, considerando que la disrupción o el conflicto son condiciones presentes.

También se reconoce la necesidad de abandonar prácticas tradicionales caracterizadas como altamente punitivas para establecer el orden y el silencio en el aula. Esto procedería del reconocimiento del maltrato o de la violencia que dichas prácticas implicaban y de la progresiva conciencia que se toma del estudiante como sujeto de derechos.

\subsubsection{Desarrollo de habilidades socio-emocionales}

Un adecuado desarrollo socioemocional y de la capacidad de relacionarse de manera favorable y funcional con los estudiantes es una característica valorada y los programas formativos deberían jugar un importante rol en que todo docente las haya desarrollado al egresar. Resulta un requisito relevante para todo docente en su práctica profesional por cuanto forma parte indisociable e indispensable de su quehacer profesional y encontrarse alineado con políticas de alcance nacional.

La habilidad más nombrada es la empatía, definida por los entrevistados como la capacidad de interesarse de una manera genuina en los escolares y acercarse a su realidad de vida y así comprender las causas profundas de eventuales problemas de comportamiento o comprensión. Gracias al despliegue de esta competencia, no sería tan necesario recurrir a decisiones superficiales y medidas punitivas. Esta competencia cobra más relevancia en contextos de desempeño profesionales con estudiantes altamente vulnerables, de realidades sociales muy distintas a los orígenes de los docentes y que requieren de manejos pedagógicos más complejos.

\subsubsection{Trabajo colaborativo entre docentes y otros adultos}

El trabajo conjunto del docente con otros adultos de los establecimientos constituye otro desafío formativo en CE. Proponen tomar distancia de una concepción individualista del rol docente. Aprecian que la dinámica de los colegios favorece la incorporación de más de un docente en el aula y a más profesiones colaborando; la capacidad de trabajar junto a los pares profesionales incluso haciendo co-docencia resulta ser deseable, tanto por su funcionalidad en relación con la enseñanza del currículum disciplinar como por su potencial modelador para la colaboración entre estudiantes.

Esta lógica de colaboración no se agota entre quienes trabajan al interior de la escuela, sino que se extendería a las relaciones que se establecen entre la escuela y las familias de los estudiantes. 


\subsection{ESTRATEGIAS CURRICULARES PARA LOS DESAFÍOS FORMATIVOS}

Esta segunda gran categoría agrupa las estrategias que los entrevistados consideran necesarias para que el proceso de innovación instale la CE como una dimensión relevante de la FID.

\subsubsection{Sistematicidad del diseño}

La innovación curricular propuesta debe resultar de un proceso de reflexión e intervención llevado a cabo en cada universidad de manera gradual y racional por los formadores de formadores. Debe ser planificada, incluyendo etapas de diagnóstico, diseño e implementación de propuestas, evaluación de impacto y considerando a la vez la participación de los actores convocados.

Existe la conciencia de que los procesos de innovación curricular son lentos y que el impacto en el ejercicio de la docencia en el medio escolar también es lento y complejo de evaluar. Además, debe tenerse en cuenta el principio de contextualización de la innovación, sobre todo si paralelamente, se considera la autonomía curricular universitaria.

\subsubsection{Articulación institucional entre niveles del sistema de Formación Inicial Docente}

La formación en CE requiere de una fluida articulación de la FID como sistema. Particularmente, la comunicación entre las instancias de diseño de políticas, las casas de estudios superiores y los colegios que funcionan como centros de práctica profesional debe ser permanente y bidireccional.

De las instancias de diseño, se espera que favorezcan el uso efectivo de instrumentos normativos que aseguren la incorporación de la CE en la FID. La idea de que los futuros docentes reconozcan esta dimensión y la importancia de prepararse profesionalmente en esta elevándola a la categoría de saber pedagógico propiamente tal. Asimismo, se espera que la Universidad conozca la realidad de sus centros de práctica, tanto en relación con el clima de aula como con la composición demográfica o psicosocial de las comunidades, para que los planes de formación sean más contextualizados y pertinentes.

\subsubsection{La tensión metodológica entre "cursificación” y "transversalización"}

Aparecen visiones metodológicas encontradas: incorporar la CE en asignaturas específicas relativas al tema o bien convertirla en una temática transversal al programa de formación.

La primera opción, referida mediante el neologismo "cursificación", significa crear asignaturas de formación pedagógica específicamente diseñadas para revisar los contenidos y habilidades señalados más arriba. La ventaja sería asegurar que todo docente haya seguido cursos de CE y que las competencias sean evaluadas y certificadas formalmente. La desventaja sería el encapsulamiento de este conocimiento en una sola asignatura y permanecer como un área del saber pedagógico desconectada de las demás.

La segunda opción, nombrada como "transversalización", se refiere a considerar la CE como un saber pedagógico íntimamente ligado a la didáctica y por lo tanto incorporable a cualquier asignatura. La ventaja sería considerar el carácter integral de la CE y la potencialidad para que sus enfoques dialoguen con los distintos saberes pedagógicos. La 
desventaja sería el peligro de ser relegada para el final o incluso omitida de las distintas asignaturas, dado que son las otras áreas del saber pedagógico las que tienen mayor relevancia histórica y culturalmente.

Con todo, se habla de optar por modelos mixtos que tomen elementos de ambas opciones: la idea de implementar cursos no es incompatible con la idea de incorporar la temática en los programas de otras asignaturas, en actividades extracurriculares o en los perfiles de egreso.

\subsubsection{Formación práctica reflexiva sobre Convivencia Escolar}

También se posiciona como un desafío vincular la formación en CE con la formación práctica. Esta recomendación tiene que ver con el reconocimiento de la potencia que tiene articular los conocimientos con lo que sucede en la realidad de las escuelas.

No se trata solamente de promover una ejercitación de las competencias desarrolladas en el aula de Pedagogía, sino de intencionar las experiencias prácticas de tal manera que se generen en ella aprendizajes nuevos, cualitativamente distintos y más potentes e integradores. La idea es tomar los ejercicios prácticos como instancias que favorezcan la reflexión en torno a los saberes pedagógicos y particularmente, aquellos relacionados con la $\mathrm{CE}$.

Además, promover distintas prácticas en contextos distintos podría ser un ejercicio recomendable para contrastar las competencias entrenadas en clases, en términos de su viabilidad y pertinencia. Esto sobre todo considerando la diversidad de contextos del ejercicio docente y las distintas dimensiones de vulnerabilidad que caracterizan a muchos de ellos.

\subsubsection{Mecanismos de evaluación}

El desafío identificado consiste en consensuar un sistema de evaluación de las competencias ligadas a la CE tanto para las asignaturas como para las pre-prácticas y prácticas profesionales finales. Para esto, lo que se propone es tomar el lenguaje usado en el MBE, tal como se han ido alineando las demás políticas públicas en educación, para hacer uso de referentes comunes.

También se señala que la evaluación debe ser continua a lo largo de las experiencias prácticas y no sólo al final, sino que debe dar cuenta del progreso del estudiante. Asimismo, los criterios no deben ser conocidos sólo al interior de las universidades sino también en los centros de práctica.

\subsection{TRANSFORMACIONES CULTURALES}

Esta tercera gran categoría agrupa desafíos de carácter cultural, los que deben dar un sustento a los desafíos de carácter más operativo señalados más arriba.

\subsubsection{Valorar la Convivencia Escolar en la cultura institucional de la Formación Inicial Docente}

Un gran desafío tiene que ver con las condiciones subjetivas en que el posicionamiento de la CE se puede dar al interior de las carreras de Pedagogía. En este sentido, no basta 
con la incorporación de las distintas temáticas o dimensiones de la CE a los programas: la modernización de las propuestas formativas no debe sustentarse en una mera aceptación de los lineamientos de la política pública, sino en una toma de conciencia profunda por parte de los formadores de formadores respecto de la importancia de la CE en el ejercicio de la docencia.

En concordancia con lo anterior, se refuerza la idea de que la incorporación de elementos de CE al currículum de las Pedagogías no debe ser ni una decisión opcional ni menos un sello valórico que distinga a ciertas universidades. Debe empezar a entenderse por los formadores de formadores como un requisito profesional.

\subsubsection{Considerar la Convivencia Escolar como parte esencial de la identidad profesional docente}

Es preciso tomar conciencia que la CE forma parte esencial del oficio docente y, por lo tanto, las competencias asociadas son parte constitutivas de la identidad profesional docente. Las responsabilidades profesionales en torno a la $\mathrm{CE}$ deben ser asumidas desde el principio de la FID, tomando distancia de una mirada tradicional que las considere un apéndice del rol.

Se ha planteado que el atender a elementos relacionales ya sea al interior del estamento estudiantil o en los equipos profesionales, sería una tarea de la que se han apropiado más fácilmente docentes de niveles como el Pre-Básico, Básico o Diferencial, en contraposición a docentes de Enseñanza Media, incluyendo a los docentes de la modalidad Técnico Profesional, cuyos programas de formación se centran mucho más sus disciplinas. No obstante, se reconoce que, en el cotidiano, siempre será parte del oficio el atender a necesidades relacionales o psicosociales de los estudiantes.

\section{DISCUSIÓN Y CONCLUSIONES}

El primer conjunto de resultados hace referencia a los desafíos formativos en CE que enfrenta la FID, señalando contenidos, habilidades o competencias que deberían ser objeto del proceso de enseñanza-aprendizaje de futuros docentes. Es posible afirmar que las categorías propuestas (las herramientas conceptuales, la diversidad, la violencia y el conflicto, el clima de aula, las habilidades socioemocionales y el trabajo colaborativo con adultos) tienden a coincidir más con las demandas de formación relevadas por los propios docentes en investigaciones anteriores (Ruffinelli, 2013; Gaete, Gómez y Bascopé, 2016) que con las distintas dimensiones que componen el concepto amplio de $\mathrm{CE}$, el que considera otras dimensiones como la formación en valores, para la participación democrática y ciudadana o la comprensión del sistema educacional y las políticas públicas del área (García, 2008; Canedo, 2014). Podría sostenerse que los desafíos propuestos se construyen más a partir de necesidades prácticas de la docencia que del desarrollo teórico-conceptual de la CE y de las políticas públicas propuestas (MINEDUC, 2002, 2003, 2015 y 2019). Lo anterior podría deberse a que, por una parte, las políticas públicas en materia de CE no han tenido suficiente centralidad en la política educativa y por otra, a que la perspectiva que surge desde la práctica docente no ha logrado ser incorporada en la política pública y en los espacios formativos. Lo anterior implica la necesidad de rescatar, con algún criterio educativo, los requerimientos que surgen de ambos niveles del sistema. 
Algunos de los desafíos formativos merecen un mayor análisis:

En relación con la formación conceptual en CE, llama la atención el énfasis otorgado a la formación en psicología que manifiestan los entrevistados. El relacionar las complejidades de la CE con las características individuales de los niños y jóvenes concuerda con lo que López et al. (2011) cuando se refieren al proceso de individualización como explicación de los fenómenos de violencia y por extensión, de la CE. La implicancia de lo anterior es que esto podría excluir las numerosas dimensiones conceptuales descritas tanto en la investigación y las políticas de CE que son sintetizadas por García (2008) y por Canedo (2014) y que se refieren, entre otras temáticas, a la inclusión, el aprendizaje de la diversidad, la promoción de la mediación, la recuperación del diálogo entre la escuela y la familia, la participación, la formación en valores y el desarrollo socioemocional entre otras.

Lo mencionado se relaciona con la necesidad de considerar la gestión pedagógica de la diversidad. Esto se instala como un desafío interesante que se relaciona tanto con lo planteado por el MBE (MINEDUC, 2013) como por el requerimiento de inclusión escolar.

Por otra parte, la necesidad de contar con un clima de aula adecuado con el fin de favorecer el desarrollo de clases eficaces refuerza lo planteado por Valdivieso et al. (2017) respecto de que la CE se instala como un medio que permite el logro de una buena enseñanza. Sin perjuicio de lo anterior, la dimensión de la CE como un fin planteada por los autores no aparece relevada.

La valoración de respecto del desarrollo de habilidades socio-emocionales en los futuros profesores es coherente con la literatura en la materia, especialmente con lo propuesto por Canedo (2014), quien releva dicha dimensión.

Finalmente, llama la atención que surja como desafío formativo el aprendizaje del trabajo colaborativo entre adultos como una necesidad ligada al desarrollo de la CE. Permite ampliar el concepto a las relaciones entre los adultos y de la escuela con las familias, lo que es coherente con las necesidades rescatadas por Ruffinelli (2013) y Gaete, Gómez y Bascopé (2016).

La segunda dimensión dice relación con aquellas estrategias curriculares que se requieren para implementar los desafíos formativos en CE que tiene la FID. Una primera categoría propone que la necesidad de la construcción curricular progresiva, sistemática, reflexiva y pertinente en contenidos y habilidades en CE.

Para lo anterior, se requiere que los diferentes niveles del sistema escolar acuerden visiones e instrumentos normativos que aseguren que la $\mathrm{CE}$ sea una dimensión reconocida y validada de manera formal en el sistema de formación. Esto sigue la línea descrita por autores como Donoso (2008), Sotomayor y Gysling (2011), Ahumada y Tapia (2013) y Valdivieso et al. (2017) relativa a la necesidad de una progresiva institucionalización de la CE en las políticas públicas.

Una discusión muy relevante es aquella que instala la pregunta respecto de si estos conocimientos y habilidades docentes deben ser instalados mediante cursos especializados o de manera transversal. Al respecto, habría que pensar que esta dicotomía, expresada a propósito de transversalidad educativa (MINEDUC, 2012 y 2013) debiese ser resuelta a favor de un modelo mixto que incluya, tanto cursos relativos a las temáticas de CE, como la transversalización de los mismo en la enseñanza de las diferentes áreas de la FID. Lo relevante será que dicha formación tenga un importante componente reflexivo que favorezca el diálogo entre la teoría y la práctica docentes con los objetivos políticos y éticos de la educación (Gomes \& Pereira, 2009; Pérez, 2018). 
Asimismo, los procesos formativos en CE requieren de mecanismo de evaluación de estos, lo que se corresponde con lo planteado por autores como Ávalos $(2004,2014)$ y Baeza et al. (2006), quienes proponen que el MBE también sería un referente para los efectos de evaluación.

Contribuyendo a esta idea, se posiciona la necesidad de favorecer condiciones culturales que permitan el posicionamiento de las temáticas de CE en la FID. El desafío señalado supera propuestas revisadas anteriormente en la medida que se limitaban a sugerir incorporación de aspectos curriculares a la FID (García, 2008; Ruffinelli, 2013; Canedo, 2014; Gaete, Gómez y Bascopé, 2016), apuntando a cambios en la propia cultura institucional de las universidades formadoras de docentes. De lo que se trata es lograr concientizar respecto de la CE como un aspecto esencial de la labor docente cotidiana y, por tanto, de la identidad profesional del gremio (Cox y Gysling, 2009).

La idea propuesta de enfocar la comprensión de la CE en la escuela y en la FID también como una finalidad de la educación (Valdivieso et al., 2017) podría encontrar un referente en el caso de España, en que se ha decidido explicitar que los maestros se forman y se desempeñan profesionalmente en el compromiso con la democracia. El enriquecimiento de la FID para que la identidad profesional incorpore el compromiso con la CE como un fin en sí mismo, para el caso de Chile, debería relacionarse directamente con el compromiso con el aseguramiento de la paz y de la democracia, buscando oponerse a un retroceso autoritario (Magendzo et al., 2013), horizonte que todavía no se considera.

En síntesis, este estudio permite concluir que el principal desafío (y que engloba los demás) consiste en enriquecer el currículum de la FID de manera tal que habilite a los docentes para hacerse cargo profesionalmente de la dimensión convivencial de la escuela. Se han visibilizado desafíos formativos y estrategias curriculares para su incorporación, como también desafíos que trascienden la FID entendida como currículum, proponiendo favorecer en los propios formadores de formadores la valoración de la CE como parte esencial del oficio y de la identidad profesional docente, como sustento cultural del proceso. Lo anterior permitirá avanzar en la dirección señalada por Arístegui et al. (2005) respecto a la relevancia de formar a los futuros maestros en una pedagogía de la convivencia.

Se propone que futuras investigaciones consideren a los nuevos formadores de formadores llamados "mentores" en el marco del Sistema Nacional de Desarrollo Profesional Docente (pues no se encontraban todavía en actividad durante la etapa de producción de datos). Otros informantes claves podrían ser los estudiantes de Pedagogía o los estudiantes escolares, con el objeto de diversificar las miradas que aportan al desarrollo de la FID. También se hace necesario profundizar la comprensión de los vínculos entre identidades profesionales docentes, los desafíos al ejercicio docente y la CE, con el fin de favorecer la pertinencia de la innovación curricular en las Pedagogías. En la misma lógica, el tema de esta investigación no se agota en la FID y futuras investigaciones podrían estudiarlo en otras etapas de la carrera profesional.

\section{REFERENCIAS BIBLIOGRÁFICAS}

Ahumada, I. y Tapia, J. (2013). Principales características y contenidos de la política educacional chilena sobre Convivencia Escolar entre los años 2002 y 2012: Una aproximación a la realidad 
del sistema educacional chileno a partir de un análisis de contenido temático. (Memoria de título). Universidad de Chile, Santiago, Chile.

Arístegui, R., Bazán, D., Leiva, J., López, R., Muñoz, B., y Ruz, J. (2005). Hacia una Pedagogía de la Convivencia. Psykhe (Santiago), 14(1), 137-150.

Ávalos, B. (2014). Initial teachers' education in Chile: tensions between policies of support and control. Estudios pedagógicos (Valdivia), 40(Especial), 11-28

(2004). La formación docente inicial en Chile. Digital observatory for higher education in Latin America and the Caribbean. UNESCO, IESALC. Santiago de Chile.

Baeza, J., Pérez, M. y Reyes, L. (2006). Estándares de Desempeño Docente. Sistematización de Contenidos para su operacionalización. Ediciones Universidad Católica Silva Henríquez.

Canales, M., Bellei, C., y Orellana, V. (2016). ¿Por qué elegir una escuela privada subvencionada? Sectores medios emergentes y elección de escuela en un sistema de mercado. Estudios Pedagógicos (Valdivia), 42(3), 89-109.

Canedo, D. (2014). Conocimientos y habilidades relativas a la convivencia escolar presentes en la formación inicial docente. (Tesis de pregrado). Universidad de Chile, Santiago, Chile.

Carrasco, C., López, V. y Estay, C. (2012). Análisis Crítico de la Ley de Violencia Escolar de Chile. Psicoperspectivas, 11(2), 31-55.

Cisternas, T. (2011). La investigación sobre formación docente en Chile: Territorios explorados e inexplorados. Calidad en la educación, (35), 131-164.

CNA Chile (2018). Santiago. Recuperado de https://www.cnachile.cl/noticias/Paginas/SECONSOLIDAN-LAS-PEDAGOG\%C3\%8DAS.aspx

Contreras, C. (2014) El desarrollo docente del formador de profesores: una propuesta orientada hacia el análisis de incidentes críticos auténticos. Estudios Pedagógicos, XL, (Especial), 49-69.

Cornejo, R. (2008). Entre el sufrimiento individual y los sentidos colectivos: Salud laboral docente y condiciones de trabajo. Docencia, 12(35), 77-85.

Cox, C. y Gysling, J. (2009), La formación del profesorado en Chile. 1842 - 1987. Ediciones Universidad Diego Portales, Santiago de Chile.

Decreto Supremo 381 del Ministerio de Educación. Biblioteca del Congreso Nacional de la República de Chile. Santiago, Chile (10 de julio de 2013).

Delors, J. (1996). La educación encierra un tesoro. Informe a la UNESCO de la Comisión Internacional sobre la educación para el siglo XXI. Madrid: Santillana/UNESCO.

Donoso, S. (2008). El perfeccionamiento docente en Chile (1990-2007): ¿estado versus mercado? Revista Brasileira de Educação, 13(39), 437-454.

Ferrada, D., Villena, A. y Turra, O. (2015). Transformación de la formación. Las voces del profesorado. Santiago: RIL Editores.

Gaete, A., Gómez V., y Bascopé, M. (2016). ¿Qué le piden los profesores a la formación inicial docente en Chile? Temas de la agenda pública, (86).

García, L. (2008). La Convivencia como recurso educativo. Hacia el diseño de un Plan de Convivencia para los centros docentes de la Comunidad Valenciana. (Tesis doctoral). Universitat de Valencia, Valencia, España.

Gomes, C. \& Pereira, M. (2009). Teacher education in face of violence from/at schools. Cadernos de Pesquisa, 39(136), 201-224.

Gómez, H. (2016). Formación Docente: Demandas desde la frontera. Santiago: UCSH.

Ley N 20.536 sobre Violencia Escolar. Biblioteca del Congreso Nacional de la República de Chile. Santiago, Chile (8 de septiembre de 2011).

Leyton, I. (2014). Revisión de contenidos relativos al desarrollo de conocimientos y habilidades de Convivencia Escolar en la Formación Inicial Docente de educación media en dos universidades de la Región Metropolitana. (Tesis de maestría). Universidad de Chile, Santiago, Chile.

López, V., Carrasco, C., Morales, M., Ayala, A., López, J. y Karmy, M. (2011). Individualizando la Violencia Escolar: Análisis de Prácticas Discursivas en una Escuela Municipal de la 
Región de Valparaíso. Psykhe (Santiago), 20(2), 7-23. https://dx.doi.org/10.4067/S071822282011000200002

Magendzo, A., Toledo, M., y Gutiérrez, V. (2013). Descripción y análisis de la Ley sobre Violencia Escolar ( $\left.{ }^{\circ} 20.536\right)$ : dos paradigmas antagónicos. Estudios pedagógicos (Valdivia), 39(1), 377391. https://dx.doi.org/10.4067/S0718-07052013000100022

Manzi, J. (2010). Programa INICIA: fundamentos y primeros avances. Ecos de la revolución pingüina. Avances, debates y silencios en la reforma educacional, 285-308.

Mena, I. y Valdés, A. (2008). «Clima social escolar». Documento Valoras UC. Santiago: Escuela de Psicología, Universidad Católica.

Milicic, N. y Aron, A. M. (2000). Climas sociales tóxicos y climas sociales nutritivos para el desarrollo personal en el contexto escolar. Revista Psykhe, 9(2), 117-123.

MINEDUC. (2019). Política Nacional de Convivencia Escolar. Santiago, Chile: Ministerio de Educación.

. (2015). Política Nacional de Convivencia Escolar (2015-2018). Santiago, Chile: Ministerio de Educación.

. (2014). Otros indicadores de calidad educativa. Santiago: Ministerio de Educación de Chile.

(2013). Bases Curriculares 7mo básico a 2do medio. Santiago: Ministerio de Educación,

Gobierno de Chile.

. (2012). Bases Curriculares para la Educación Básica. Santiago: Ministerio de Educación,

Gobierno de Chile.

. (2009). Objetivos Fundamentales y Contenidos Mínimos Obligatorios de la Educación Básica y Media. Santiago: MINEDUC.

. (2006). Ley No 20.129 Del Sistema de Aseguramiento de la Calidad de la Educación Superior. Santiago: MINEDUC.

(2003). Marco Para La Buena Enseñanza. Santiago: MINEDUC.

(2002). Política de Convivencia Escolar de Chile. Santiago: Unidad de Apoyo a la Transversalidad, Ministerio de Educación, Gobierno de Chile.

Miranda, C., y Rivera, P. (2009). Formación permanente de profesores: ¿Quién es el formador de formadores? Estudios pedagógicos (Valdivia), 35(1), 155-169.

Pérez, T. (2018). Maestros para la democracia. La formación inicial del magisterio en España con la Ley de Ordenación General del Sistema Educativo (1990). História da Educação, 22(54), 299-317.

Reyes-Jedlicki, L., Miranda, C., Santa Cruz, E., Cornejo, R., Núñez, M., Arévalo, A. y Hidalgo, F. (2014). Subjetividades y saberes docentes en el sistema educativo chileno: un análisis desde las concepciones de formadores de profesores. Estudios pedagógicos (Valdivia), 40(Especial), 183-203.

Ruffinelli, A. (2013). La calidad de la formación inicial docente en Chile: la perspectiva de los profesores principiantes. Calidad en la educación, (39), 117-154.

Ruz, J. (Ed.) (2006). Convivencia y calidad de la Educación. Santiago: Organización de Estados Iberoamericanos para la Ciencia y la Educación (OEI).

Sandoval, M. (2014). Convivencia y clima escolar: claves de la gestión del conocimiento. Última década, 22(41), 153-178.

Sierra, A. (2016). Contributions of a social justice language teacher education perspective to professional development programs in Colombia. Profile Issues in Teachers' Professional Development, 18(1), 203-217.

Sotomayor, C. y Gysling, J. (2011). Estándares y regulación de calidad de la formación de profesores: discusión del caso chileno desde una perspectiva comparada. Calidad en la educación, (35), 91-129.

Strauss, A y Corbin, J. (2002). Bases de la investigación cualitativa. Técnicas y procedimientos para desarrollar la teoría fundamentada. Medellín: Contus.

Taylor, S.J. y Bogdan, R. (2000). Introducción a los métodos cualitativos de investigación. La búsqueda de significados. Barcelona: Paidós. 
Toledo, F. (2018). Concepciones sobre la violencia en el ámbito de la comunidad escolar presentes en Chile en las políticas públicas y en la literatura especializada en el nuevo milenio. (Tesis de maestría). Universidad de Chile, Santiago, Chile.

Valdivieso, P., González, P., Toledo, F. y Leyton, I. (2017). Convivencia Escolar desde la política educativa chilena ¿una finalidad de la educación? Revista Enfoques Educacionales, 14(1), 89-110. 
